\title{
Effect on survival of local treatment in patients with low prostate-specific antigen, high Gleason score prostate cancer: a population-based propensity score-matched analysis
}

\author{
Shuai Liu ${ }^{1 \#}$, Xiao-Ying Wang ${ }^{2 \#}$, Tian-Bao Huang ${ }^{3}$, Quan Niu ${ }^{4}$, Kai Yao ${ }^{3}$, Xing Song ${ }^{1}$, Shi-Yao Zhou ${ }^{1}$, \\ Zhen Chen ${ }^{1}$, Guang-Chen Zhou ${ }^{3}$ \\ ${ }^{1}$ Department of Graduate School, Dalian Medical University, Dalian, China; ${ }^{2}$ Department of Anesthesiology, ${ }^{3}$ Department of Urology, Subei \\ People's Hospital of Jiangsu Province (Clinical Medical College, Yangzhou University), Yangzhou, China; ${ }^{4}$ Department of Urology, Yiwu Municipal \\ Central Hospital, Yiwu, China \\ Contributions: (I) Conception and design: S Liu; (II) Administrative support: GC Zhou; (III) Provision of study materials or patients: S Liu; \\ (IV) Collection and assembly of data: S Liu, XY Wang, TB Huang, K Yao; (V) Data analysis and interpretation: S Liu, XY Wang, Q Niu, X Song, SY \\ Zhou, Z Chen; (VI) Manuscript writing: All authors; (VII) Final approval of manuscript: All authors. \\ "These authors contributed equally to this work. \\ Correspondence to: Guang-Chen Zhou. Department of Urology, Subei People’s Hospital of Jiangsu Province (Clinical Medical College, Yangzhou \\ University), Yangzhou, China. Email: guangchenzhou1@163.com.
}

Background: Emerging data suggest that in patients with low prostate-specific antigen (PSA) and high Gleason score, prostate cancer (PCa) is more aggressive and more likely to be related to genomic characteristics of neuroendocrinology. However, the evidence for the advantages of local treatment (LT) for these men is lacking. Hence, we investigated survival in men with low-PSA values and high-grade (Gleason score 8-10) PCa according to the treatment of the primary tumor.

Methods: Kaplan-Meier survival analysis was applied to analyze the effects on overall survival (OS) and cancer-specific survival (CSS) according to the different treatments of the primary tumor. Multivariable Cox proportional hazards survival regression analysis calculated the CSS after propensity score matching (PSM) in 2 cohorts according to treatment type. The treatment types included the following: (I) LT versus non-LT (NLT) and (II) radical prostatectomy (RP) versus radiotherapy (RT).

Results: In the Surveillance, Epidemiology, and End Results (SEER) database [2004-2014], we identified 14,208 patients newly diagnosed with low PSA values (10 ng/mL or less), with a Gleason score 8-10, and cT1-4N0M0 prostate adenocarcinoma. After the first PSM, of the 3,512 PCa patients, 1,576 underwent LT and 1,576 underwent NLT. After the second PSM, of the 792 PCa patients, 396 underwent RP, and 396 underwent RT. The 5- and 10-year OS rates for LT vs. NLT patients were $90 \%$ and $73 \%$ versus $69 \%$ and $39 \%$, respectively, while the 5- and 10-year CSS rates for LT vs. NLT patients were $98 \%$ and $94 \%$ versus $89 \%$ and $79 \%$, respectively. Subsequent multivariate survival analysis showed that LT was associated with lower likelihood of PCa mortality relative to NLT [hazard ratio (HR), 0.19; 95\% confidence interval (CI), 0.14-0.26, $\mathrm{P}<0.001$ ], also in RP versus RT (HR, 0.26; 95\% CI, 0.12-0.54, $\mathrm{P}<0.001$ ).

Conclusions: In patients with low PSA values, Gleason score 8-10, and localized PCa, LT resulted in higher survival compared with NLT. Within LT, RP provided the most benefit relative to RT.

Keywords: Prostate cancer (PCa); radical prostatectomy (RP); radiotherapy (RT); non-local treatment (NLT); prostate-specific antigen (PSA); propensity score matching (PSM)

Submitted Oct 23, 2019. Accepted for publication Apr 07, 2020.

doi: 10.21037/apm-19-414

View this article at: http://dx.doi.org/10.21037/apm-19-414

(c) Annals of Palliative Medicine. All rights reserved. 


\section{Introduction}

Prostate cancer (PCa), mainly prostate adenocarcinoma, is one of the most frequent malignancies in males in the United States (US), and the second leading cause of cancer mortality for men in the US (1). Widespread use of prostatespecific antigen (PSA) screening has led to a reducing of PCa's mortality (2). However, many patients with $\mathrm{PCa}$ are asymptomatic, and clinically evident symptoms will not emerge during the patient's lifetime (3). The extensive use of the PSA test as a screening tool has led to the increase in early diagnosis of $\mathrm{PCa}$, and has also enhanced treatment rates for $\mathrm{PCa}$ (4). However, the value of PSA screening has continued to be debated. For instance, the US Preventive Services Task Force (USPSTF) published a guideline in 2012 recommending against PSA screening in all men, but then, in 2018, advised men aged 55-69 years to discuss the risks and benefits of screening with their healthcare providers (5).

Although PSA usually rises in high tumor grades, some high-grade but poorly differentiated cancers may be associated with low PSA production (6). The National Comprehensive Cancer Network (NCCN) guidelines (7) do not recommend routine prostate biopsy for patients with normal $(0-4 \mathrm{ng} / \mathrm{mL})$ or a gray area $(4-10 \mathrm{ng} / \mathrm{mL})$ PSA levels. Unfortunately, some studies suggest that patients with a Gleason score of $8-10$ but a low PSA level may have the most aggressive form of $\mathrm{PCa}$, which may respond poorly to treatments (8-10). In these patients with localized PCa, both the European Association of Urology (EAU) (11) and NCCN guidelines (12) recommended local treatment (LT), which includes radical prostatectomy (RP) and radiotherapy (RT). Although some studies have reported benefits for LT versus NLT in clinically localized PCa, the influence of LT versus NLT on cancer-specific survival (CSS) in men with low PSA values, high Gleason scores, and localized PCa is unknown. We hypothesized that men with a high Gleason score (8-10), low PSA values (10 ng/mL or less), and localized PCa might still benefit from primary tumordirected treatments compared to NLT.

\section{Methods}

\section{Patient selection}

The Surveillance Epidemiology and End Results (SEER) database includes 18 cancer registries that cover $28 \%$ of the U.S. population, with data related to cancer-based demographics, stage at diagnosis, primary treatment, follow-up of survival, etc. Using the latest version of the
SEER database, we focused on men with localized (cT1T4, N0, M0, PSA $10 \mathrm{ng} / \mathrm{mL}$ or less, and a Gleason score of 8-10) prostate adenocarcinoma (International Classification of Disease for Oncology (ICD-O-3) code 61.9; pathology code 8140/3) between the years 2004 and 2014. Inclusion criteria included the following: the diagnosis of $\mathrm{PCa}$ as primary cancer, available dates of survival months, active follow-up, and patients who survived for more than 1 days. Patients diagnosed with PCa before 2004 were not included because PSA information was not available in SEER before then, and thus would not allow for sufficient follow-up time to evaluate survival. PSA values in the SEER database have been audited and are now available for all patients diagnosed between 2004 and 2014 (13). We selected patients who underwent RP or brachytherapy, but external beam radiotherapy (EBRT) patients were excluded, due to lack of EBRT organ site-specific code information (14); other surgical methods except for RP, such as transurethral prostatectomy, were also excluded.

\section{Propensity score matching (PSM)}

For the PSM (1:1 ratio, with a matching tolerance of $0.0005)$, adjustment variables consisted of age, race, marital status, PSA values, Gleason score, and clinical $\mathrm{T}$ stage. PSM (1:1 ratio, with a matching tolerance of 0.0009$)$ was repeated for the RT versus RP cohort. After PSM, there were no remarkable differences in age, race, marital status, PSA values, Gleason score, and clinical $\mathrm{T}$ category in the matched cohorts (Tables 1 and 2).

\section{Statistical analyses}

Kaplan-Meier survival analysis was applied to analyze the effect on overall survival (OS) and CSS according to the different treatments of the primary tumor. The multivariable analysis of Cox's proportional hazards for survival regression was used to test the effect of LT versus NLT on CSS. P values $<0.05$ were considered statistically significant. All statistical analyzes were performed using the program SPSS version 24.0 (IBM Corporation, Armonk, NY, USA).

\section{Results}

Within the SEER database [2004-2014], we identified 14,208 patients newly diagnosed with PCa, 10,718 of whom underwent LT $(\mathrm{RP}=9,837$; RT $=881)$, and 3,490 of 
Table 1 Baseline characteristics of the 14,208 patients with non-metastatic (cT1-4, N0, M0, PSA $\leq 10$ ng/mL, and Gleason score 8-10) prostate cancer between 2004 and 2014 from the SEER database

\begin{tabular}{|c|c|c|c|c|c|c|}
\hline Variables & \multicolumn{3}{|c|}{ Local treatment } & \multicolumn{3}{|c|}{ Propensity score-matched local treatment } \\
\hline Age at diagnosis (years) & & & $<0.001$ & & & 0.71 \\
\hline$<60$ & $2,731(25.5)$ & $283(8.1)$ & & $153(9.7)$ & $170(10.8)$ & \\
\hline $60-69$ & $5,666(52.9)$ & $860(24.6)$ & & $545(34.6)$ & $531(33.7)$ & \\
\hline$\geq 80$ & $117(1.1)$ & $996(28.5)$ & & $99(6.3)$ & $106(6.7)$ & \\
\hline Race & & & $<0.001$ & & & 0.21 \\
\hline White & $8,648(80.7)$ & $2,608(74.7)$ & & $1,292(82.0)$ & $1,267(80.4)$ & \\
\hline Black & $1,291(12.0)$ & $597(17.1)$ & & $189(12.0)$ & $220(14.0)$ & \\
\hline Marital status & & & $<0.001$ & & & 0.252 \\
\hline Married & $8,224(76.7)$ & $1,878(53.8)$ & & $983(62.4)$ & $1,020(64.7)$ & \\
\hline Divorced/widowed/separated & $1,068(10.0)$ & $547(15.7)$ & & $200(12.7)$ & 208 (13.2) & \\
\hline Single & $908(8.5)$ & $321(9.2)$ & & $182(11.5)$ & $152(9.6)$ & \\
\hline Unknown & $518(4.8)$ & $744(21.3)$ & & $211(13.4)$ & $196(12.4)$ & \\
\hline Gleason score & & & $<0.001$ & & & 0.999 \\
\hline 8 & $7,269(67.8)$ & $2,233(64.0)$ & & $1,062(67.4)$ & $1,063(67.4)$ & \\
\hline 9 & 3,302 (30.8) & 1,127 (32.3) & & $485(30.8)$ & $484(30.7)$ & \\
\hline Clinical T stage & & & $<0.001$ & & & 0.353 \\
\hline $\mathrm{T} 1$ & $543(5.1)$ & $1,790(51.3)$ & & $488(31.0)$ & $524(33.2)$ & \\
\hline $\mathrm{T} 2$ & $6,283(58.6)$ & $1,571(45.0)$ & & 991 (62.9) & $954(60.5)$ & \\
\hline T3 & 3,691 (34.4) & $99(2.8)$ & & $84(5.3)$ & $79(5.0)$ & \\
\hline $\mathrm{T} 4$ & 201 (1.9) & $30(0.9)$ & & $13(0.8)$ & 19 (1.2) & \\
\hline
\end{tabular}

SEER, Surveillance, Epidemiology, and End Results; PSA, prostate-specific antigen.

whom received NLT. The characteristics of the patients are summarized in Table 1. Overall, $79.2 \%$ of patients were ethnically White, and $72 \%$ were married. For PSA values and the Gleason scores, $66.9 \%$ of patients were Gleason score $8,76.2 \%$ of patients were PSA value 4.1-10.0, while only $1.9 \%$ of the patients were Gleason score 10 , and only $15.2 \%$ patients were PSA value $\leq 2.5$. Of the 14,208 patients, $22.7 \%$ were clinical $\mathrm{T}$ stage 1 (T1), $49.0 \%$ were $\mathrm{T} 2,26.7 \%$, were $\mathrm{T} 3$, and $1.6 \%$ were $\mathrm{T} 4$.

Before PSM, it was found that NLT cohort patients 
Table 2 Baseline characteristics of the 10,718 patients with locally treated, non-metastatic (cT1-4, N0, M0, PSA $\leq 10$ ng/mL and Gleason score 8-10) prostate cancer between 2004 and 2014 from the SEER database

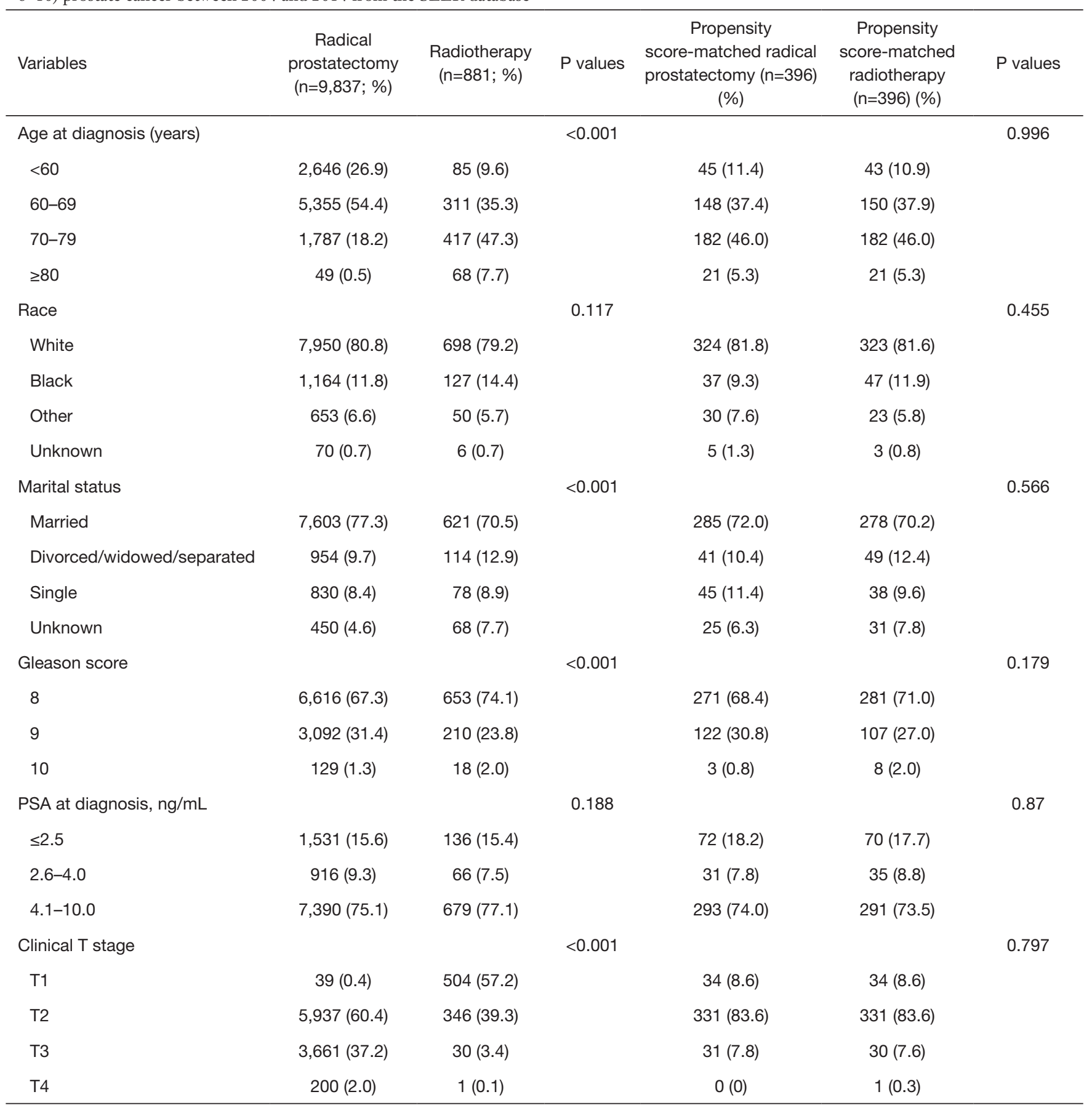

SEER, Surveillance, Epidemiology, and End Results; PSA, prostate-specific antigen.

were older (age $\geq 70: 67.2 \%$ versus $21.7 \%, \mathrm{P}<0.001$ ) and had higher Gleason scores (Gleason score $\geq 9: 36.0 \%$ versus $32.2 \%, \mathrm{P}<0.001)$ relative to the LT patients (Table 1 ). After the first PSM, of the 3,512 PCa patients, 1,576 underwent LT and 1,576 underwent NLT. After the second PSM, of the 792 PCa patients, 396 underwent RP, and 396 underwent RT. After completion of PSM, there were no remarkable differences in age, race, marital status, PSA 

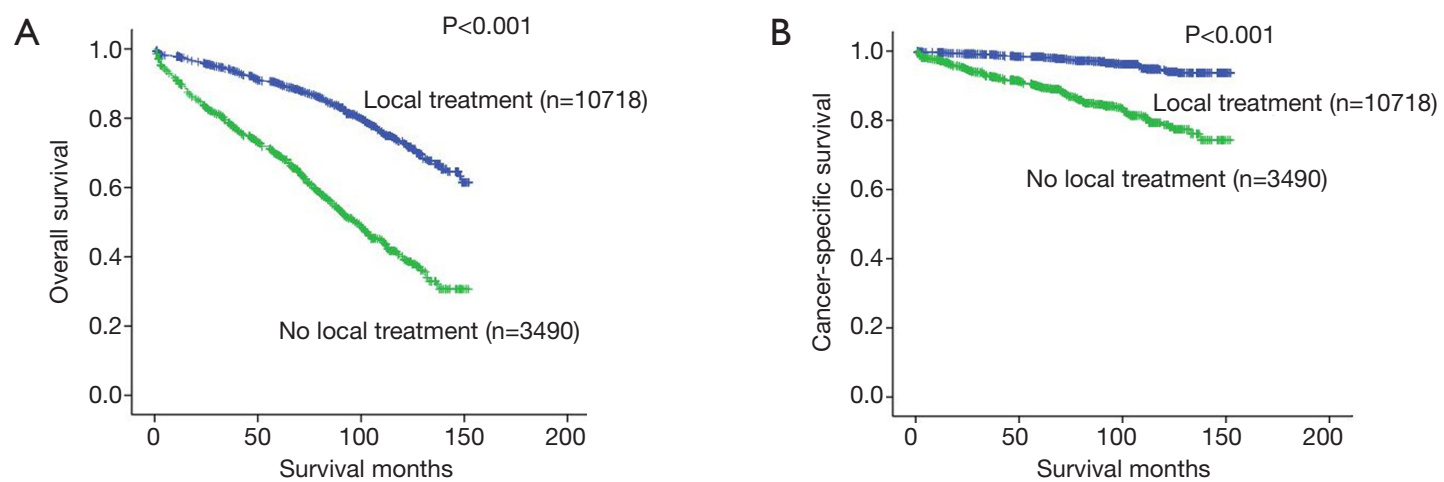

Figure 1 Kaplan-Meier survival curves of overall survival and cancer-specific survival according to treatment type.

values, Gleason score, or clinical T category in the matched cohorts (Tables 1 and 2).

The OS and CSS were calculated according to each primary cancer treatment. Kaplan-Meier survival analyses demonstrated that overall 5- and 10-year OS rates were $90 \%$ and $73 \%$ for LT patients and $69 \%$ and $39 \%$ for NLT patients, respectively $(\mathrm{P}<0.001)$ (Figure $1 A)$. Overall 5 - and 10 -year CSS rates for LT versus NLT patients were $98 \%$ and $94 \%$ versus $89 \%$ and $79 \%$, respectively, $(\mathrm{P}<0.001)$ (Figure 1B).

Analogously, the 5- and 10-year OS rates recorded for LT versus NLT patients were $92 \%$ versus $69 \%$ and $78 \%$ versus $40 \%$ for PSA values $\leq 2.5$, respectively $(\mathrm{P}<0.001)$ (Figure $2 A$ ); the 5- and 10-year OS rates recorded for LT versus NLT patients were $93 \%$ versus $62 \%$ and $69 \%$ versus $37 \%$ for PSA values $2.6-4.0$, respectively $(\mathrm{P}<0.001)$ (Figure $2 B)$; the 5 and 10 -year OS rates for LT versus NLT patients were $89 \%$ versus $69 \%$ and $72 \%$ versus $39 \%$ for PSA values $4.1-10.0$, respectively $(\mathrm{P}<0.001)$ (Figure $2 C)$; the 5 - and 10 -year CSS LT versus NLT patients were $98 \%$ versus $87 \%$ and $95 \%$ versus $74 \%$ for $\mathrm{PSA}$ values $\leq 2.5$, respectively $(\mathrm{P}<0.001)$ (Figure 2D); the 5- and 10-year CSS rates for LT versus NLT patients were $98 \%$ versus $84 \%$ and $91 \%$ versus $57 \%$ for PSA values 2.6-4.0, respectively $(\mathrm{P}<0.001)$ (Figure $2 E$ ); the 5 - and 10-year CSS rates recorded for LT versus NLT men were $98 \%$ versus $90 \%$ and $94 \%$ versus $82 \%$ for PSA values 4.1-10.0, respectively $(\mathrm{P}<0.001)$ (Figure $2 F)$.

After PSM the multivariable cox proportional hazards survival regression analysis revealed that LT was associated with a lower likelihood of PCa mortality relative to NLT [hazard ratio (HR), 0.19; 95\% confidence interval (CI), $0.14-0.26, \mathrm{P}<0.001]$, and this was also the case for RP versus $\mathrm{RT}$ (HR, 0.26; 95\% CI, 0.12-0.54, $\mathrm{P}<0.001$ ) (Tables 3 and 4).

\section{Discussion}

An elevated PSA level is a well-known high-risk factor and related to poor prognosis (12). While PSA is usually elevated in high tumor level, some men with high Gleason scores (Gleason score 8-10) and low-PSA PCa may have neuroendocrine dedifferentiated cancer cells, which may be associated with low PSA production $(6,8)$. Neuroendocrine $\mathrm{PCa}$ is a rare form of $\mathrm{PCa}$ with a worse prognosis and is associated with advanced-stage disease and resistance to castration (15). Histologically, it can be confused with partial prostate adenocarcinoma, and may also be partially transformed during treatment from prostate adenocarcinoma (9). Usually, acquired or treatment-induced neuroendocrine PCa is lethal (16).

By using large data sets of 3 separate databases and combining previous studies, one study found that patients with low PSA values and high Gleason score have neuroendocrine dedifferentiation and an aggressive, uniquely behaving form of $\mathrm{PCa}$ (9). Surgery with or without RT and/or systemic care has been shown to be a more effective initial treatment for these patients, even though the acquired and intrinsic resistance to deprivation in $\mathrm{PCa}$ is more deadly $(9,16)$.

The present study used a national contemporary cohort from the SEER database, and included only those patients with a Gleason score of $8-10$, low PSA values $(10 \mathrm{ng} / \mathrm{mL}$ or less), and non-metastatic disease for the survival analysis, yielding several new findings. First, we found that LT results in higher survival compared with NLT, which was also true for RP when compared to RT. This suggests that LT should 
A

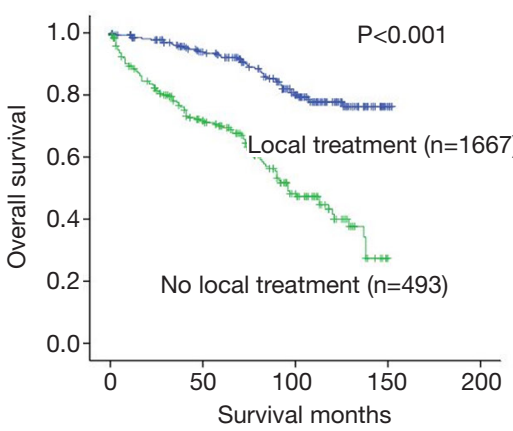

D

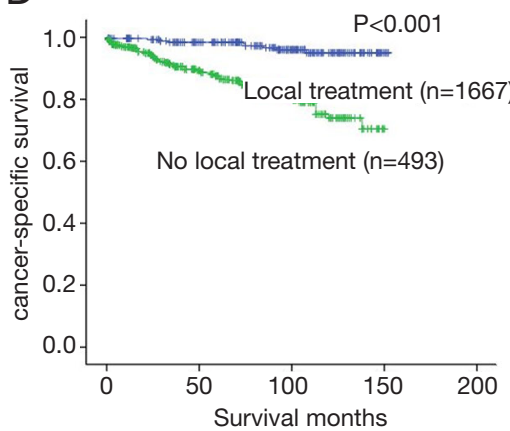

B

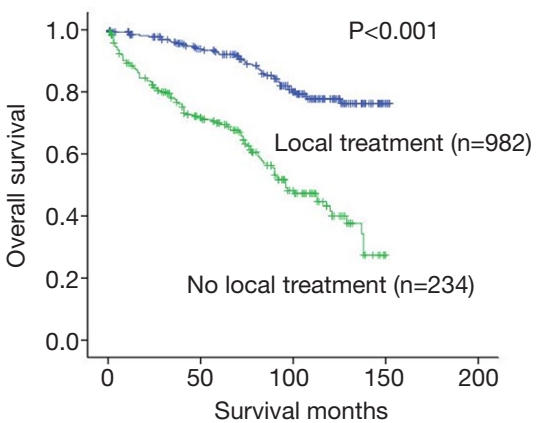

E

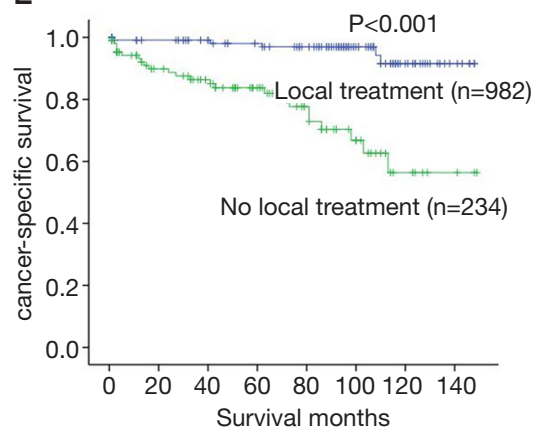

C

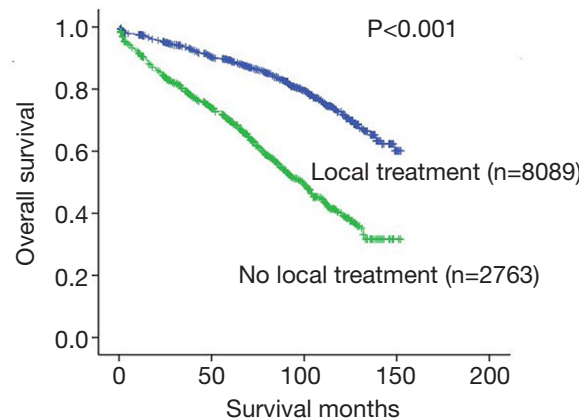

$\mathrm{F}$

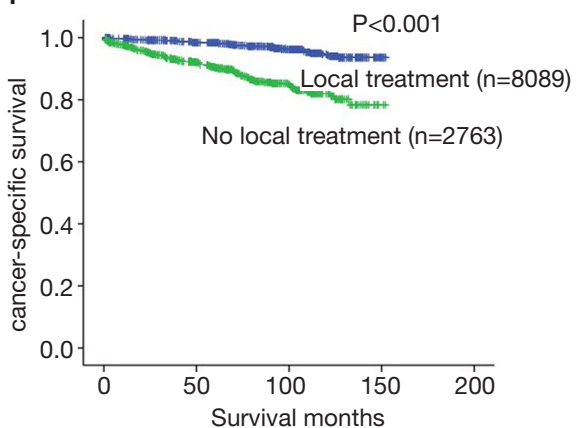

Figure 2 Kaplan-Meier survival curves of overall survival according to treatment type for PSA values (A) $\leq 2.5$, (B) 2.6-4.0, and (C) 4.1-10; and cancer-specific survival according to treatment type for PSA values (D) $\leq 2.5$, (E) 2.6-4.0, and (F) 4.1-10. PSA, prostate-specific antigen.

also be considered in patients with a Gleason score of $8-10$, low PSA values (10 $\mathrm{ng} / \mathrm{mL}$ or less), and non-metastatic PCa. We believe that these findings have the potential to help a subgroup of men who are at unusually high risk of dying from PCa. Second, stratified analyses, according to PSA values, showed higher OS and CSS rates in patients with PSA values $\leq 2.5,2.6-4.0$, and 4.1-10.0. This could support LT being considered in all PSA values, although a lower PSA value indicates a worse prognosis. Third, while some recent studies (17-20) have reported marital status to be an independent prognostic factor for $\mathrm{PCa}$, our study showed no statistical difference between different marital statuses. This discrepancy may be related to the insufficient sample size after PSM, as other studies have shown that marital quality (21) is also an important indicator of physical health. Unfortunately, the SEER database only records marital status and does not describe marital quality.

Our research is not without limitations. First, while the SEER database is an authoritative source of data to research PCa outcomes, using it involves the same inherent limitations as those of other studies based on the SEER database. Second, due to the lack of EBRT organ site- specific code information, only brachytherapy was analyzed. Third, the post-treatment PSA values or PSA doubling time information are available only for some patients, so we were not able to further analyze progression-free survival (PFS), which can better judge the efficacy of different treatment methods. Fourth, androgen-deprivation treatment (ADT) data were unavailable in the SEER database, and specific chemotherapy regimens are not provided. These therapies may influence survival in PCa patients $(9,22)$, and one hypothesis is that patients with low PSA values and a Gleason score of 8-10 might most benefit from ADT plus chemotherapy (9). In addition, the information on comorbidities, which may also influence survival in $\mathrm{PCa}$ patients (23), was unavailable in the SEER database. Because of the lack of comorbidity data, we performed multivariate analyses that controlled for confounding factors.

To conclude, as described previously, our study focused on cT1-T4 men with low PSA values and a Gleason score of 8-10 who were treated either locally or non-locally. These inclusion criteria limited study patients to a narrow range, due to the small number of the patient after PSM and with a Gleason score 10 . We were further limited by the 
Table 3 Multivariable Cox proportional hazards survival regression analysis of propensity score-matched patients diagnosed with non-metastatic (cT1-4, N0, M0, PSA $\leq 10 \mathrm{ng} / \mathrm{mL}$, and Gleason score 8-10) prostate cancer between 2004 and 2014 from the SEER database, stratified by treatment type

\begin{tabular}{|c|c|c|}
\hline Variables & \multicolumn{2}{|c|}{ Local treatment versus no local treatment (cancer-specific survival) } \\
\hline \multicolumn{3}{|l|}{ Type of therapy } \\
\hline No local treatment & Ref. & \\
\hline Local treatment & $0.19(0.14-0.26)$ & $<0.001$ \\
\hline$<60$ & Ref. & \\
\hline $60-69$ & $1.19(0.75-1.89)$ & 0.465 \\
\hline $70-79$ & $1.3(0.83-2.03)$ & 0.261 \\
\hline$\geq 80$ & $2.25(1.25-4.04)$ & 0.007 \\
\hline Black & $1.31(0.9-1.89)$ & 0.155 \\
\hline Other & $1.02(0.55-1.88)$ & 0.954 \\
\hline Unknown & - & 0.93 \\
\hline \multicolumn{3}{|l|}{ Marital status } \\
\hline Married & Ref. & \\
\hline Divorced/widowed/separated & $1.13(0.77-1.66)$ & 0.542 \\
\hline Single & $1.25(0.83-1.88)$ & 0.296 \\
\hline Unknown & $1.11(0.74-1.67)$ & 0.62 \\
\hline \multicolumn{3}{|l|}{ PSA at diagnosis, $\mathrm{ng} / \mathrm{mL}$} \\
\hline$\leq 2.5$ & Ref. & \\
\hline $2.6-4.0$ & $1.79(1.1-2.9)$ & 0.018 \\
\hline $4.1-10.0$ & $0.95(0.67-1.34)$ & 0.763 \\
\hline \multicolumn{3}{|l|}{ Clinical T stage } \\
\hline $\mathrm{T} 1$ & Ref. & \\
\hline $\mathrm{T} 2$ & $1.23(0.91-1.68)$ & 0.186 \\
\hline T3 & $1.25(0.7-2.28)$ & 0.448 \\
\hline $\mathrm{T} 4$ & 8.08 (3.86-16.89) & $<0.001$ \\
\hline
\end{tabular}

SEER, Surveillance, Epidemiology, and End Results; PSA, prostate-specific antigen; HR, hazard ratio; Cl, confidence interval; Ref., reference. 
Table 4 Multivariable Cox proportional hazards survival regression analysis of propensity score-matched patients diagnosed with non-metastatic (cT1-4, N0, M0, PSA $\leq 10 \mathrm{ng} / \mathrm{mL}$, and Gleason score 8-10) prostate cancer with local treatment between 2004 and 2014 from the SEER database, stratified by treatment type

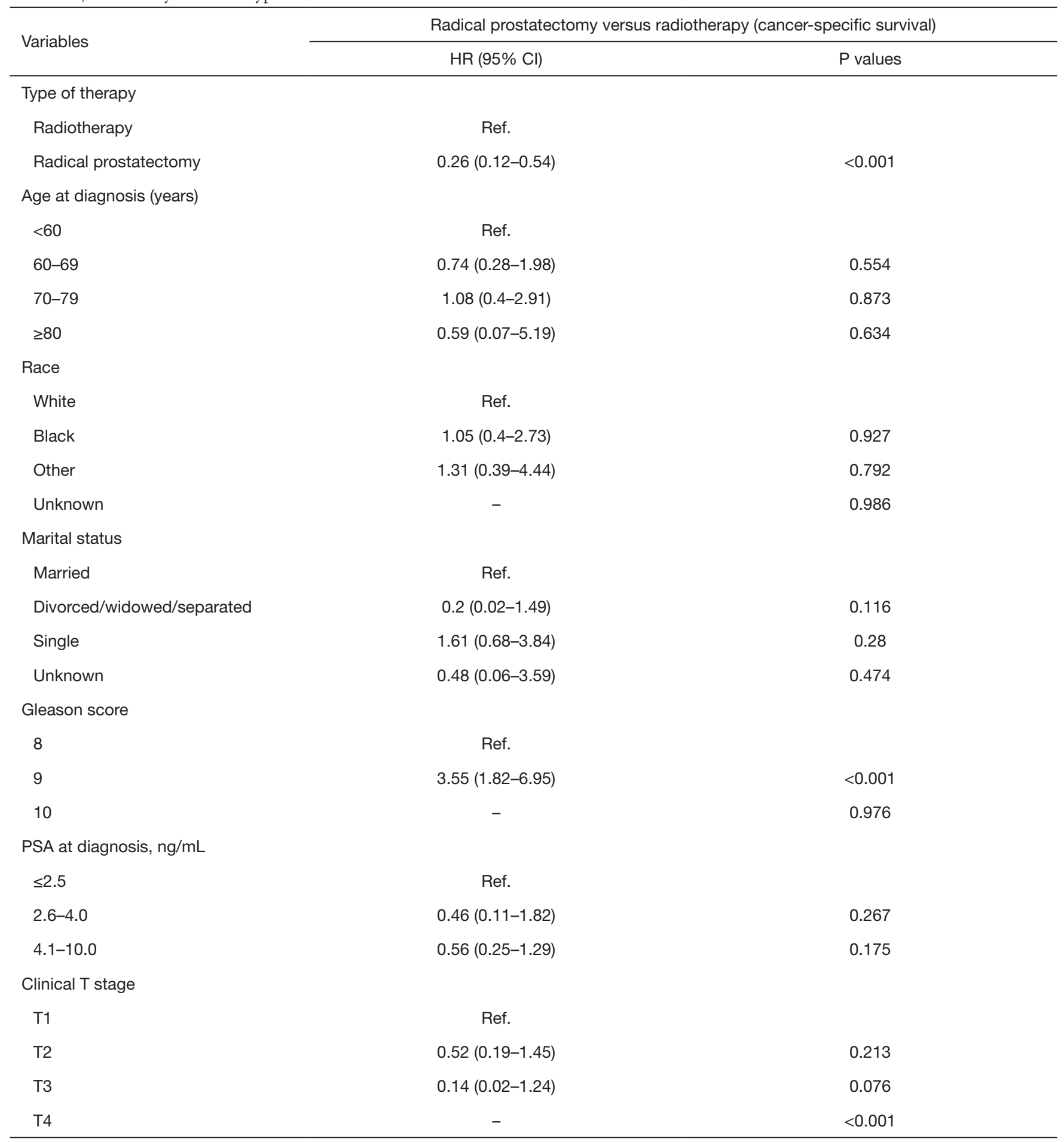

SEER, Surveillance, Epidemiology, and End Results; PSA, prostate-specific antigen; HR, hazard ratio; Cl, confidence interval; Ref., reference. 
unknown ethnic composition of the LT and NLT cohorts, and the inability to obtain HR values for clinical T4 stage patients in the RP vs. RT comparison. Nevertheless, our study still included relatively more patients and found significant and valuable results.

It is worth noting that the PSA is not a PCa specific marker, and approximately $25 \%$ of men with initial PSA values $4-10 \mathrm{ng} / \mathrm{mL}$ show PSA values upon repeat testing (24), while patients with low PSA values may also be diagnosed with PCa by biopsy (25). We urgently need new biomarkers to identify the aggressiveness of $\mathrm{PCa}$, which is not usually feasible with PSA screening as it does not exceed the typical biopsy threshold of $4.0 \mathrm{ng} / \mathrm{mL}$.

\section{Conclusions}

In conclusion, our observation provides evidence that for men with low-PSA values and high-grade (Gleason score 8-10) PCa, LT results in higher survival compared with NLT. Within LT, RP provided the most benefit relative to RT. Further research is warranted to identify the most effective treatments for these patients.

\section{Acknowledgments}

Funding: Supported by the Jiangsu Province Medical and Health research project (No. H2017060).

\section{Footnote}

Conflicts of Interest: All authors have completed the ICMJE uniform disclosure form (available at http://dx.doi. org/10.21037/apm-19-414). The authors have no conflicts of interest to declare.

Ethical Statement: The authors are accountable for all aspects of the work in ensuring that questions related to the accuracy or integrity of any part of the work are appropriately investigated and resolved. Approval was waived by the local ethics committee, as SEER data is publicly available.

Open Access Statement: This is an Open Access article distributed in accordance with the Creative Commons Attribution-NonCommercial-NoDerivs 4.0 International License (CC BY-NC-ND 4.0), which permits the noncommercial replication and distribution of the article with the strict proviso that no changes or edits are made and the original work is properly cited (including links to both the formal publication through the relevant DOI and the license). See: https://creativecommons.org/licenses/by-nc-nd/4.0/.

\section{References}

1. Siegel RL, Miller KD, Jemal A. Cancer statistics, 2019. CA Cancer J Clin 2019;69:7-34.

2. Schröder FH, Hugosson J, Roobol MJ, et al. Screening and prostate cancer mortality: results of the European Randomised Study of Screening for Prostate Cancer (ERSPC) at 13 years of follow-up. Lancet 2014;384:2027-35.

3. Grossman DC, Curry SJ, Owens DK, et al. Screening for Prostate Cancer: US Preventive Services Task Force Recommendation Statement. JAMA 2018;319:1901-13.

4. Ilic D, Djulbegovic M, Jung JH, et al. Prostate cancer screening with prostate-specific antigen (PSA) test: a systematic review and meta-analysis. BMJ 2018;362:k3519.

5. Magnani CJ, Li K, Seto T, et al. PSA Testing Use and Prostate Cancer Diagnostic Stage After the 2012 U.S. Preventive Services Task Force Guideline Changes. J Natl Compr Canc Netw 2019;17:795-803.

6. McGuire BB, Helfand BT, Loeb S, et al. Outcomes in patients with Gleason score 8-10 prostate cancer: relation to preoperative PSA level. BJU Int 2012;109:1764-9.

7. NCCN Guidelines Version 2. 2019 Prostate Cancer Early Detection. National Comprehensive Cancer Network website.

8. Mahal BA, Aizer AA, Efstathiou JA, et al. Association of very low prostate-specific antigen levels with increased cancer-specific death in men with high-grade prostate cancer. Cancer 2016;122:78-83.

9. Mahal BA, Yang DD, Wang NQ, et al. Clinical and Genomic Characterization of Low-Prostate-specific Antigen, High-grade Prostate Cancer. Eur Urol 2018;74:146-54.

10. Falchook AD, Martin NE, Basak R, et al. Stage at presentation and survival outcomes of patients with Gleason 8-10 prostate cancer and low prostate-specific antigen. Urol Oncol 2016;34:119.e19-26.

11. Mottet N, Bellmunt J, Bolla M, et al. EAU-ESTROSIOG Guidelines on Prostate Cancer. Part 1: Screening, Diagnosis, and Local Treatment with Curative Intent. Eur Urol 2017;71:618-29.

12. NCCN Guidelines Version 2. 2019 Prostate Cancer. National Comprehensive Cancer Network website.

13. Pompe RS, Tilki D, Preisser F, et al. Survival benefit of 
local versus no local treatment for metastatic prostate cancer-Impact of baseline PSA and metastatic substages. Prostate 2018;78:753-7.

14. Leyh-Bannurah SR, Gazdovich S, Budaus L, et al. Local Therapy Improves Survival in Metastatic Prostate Cancer. Eur Urol 2017;72:118-24.

15. Campedel L, Kossai M, Blanc-Durand P, et al. Neuroendocrine prostate cancer: Natural history, molecular features, therapeutic management and future directions. Bull Cancer 2017;104:789-99.

16. Fletcher CE. The Role of Splicing Regulators in the Emergence of Treatment-induced Neuroendocrine Prostate Cancer: The Next Generation of Drug Targets? Eur Urol 2019;76:167-9.

17. Huang TB, Zhou GC, Dong CP, et al. Marital status independently predicts prostate cancer survival in men who underwent radical prostatectomy: An analysis of 95,846 individuals. Oncol Lett 2018;15:4737-44.

18. Khan S, Nepple KG, Kibel AS, et al. The association of marital status and mortality among men with early-stage prostate cancer treated with radical prostatectomy: insight into post-prostatectomy survival strategies. Cancer Causes Control 2019;30:871-6.

19. Knipper S, Preisser F, Mazzone E, et al. Contemporary

Cite this article as: Liu S, Wang XY, Huang TB, Niu Q, Yao K, Song X, Zhou SY, Chen Z, Zhou GC. Effect on survival of local treatment in patients with low prostate-specific antigen, high Gleason score prostate cancer: a population-based propensity score-matched analysis. Ann Palliat Med 2020;9(4):1708-1717. doi: 10.21037/apm-19-414 analysis of the effect of marital status on survival of prostate cancer patients across all stages: A populationbased study. Urol Oncol 2019;37:702-10.

20. Liu Y, Xia Q, Xia J, et al. The impact of marriage on the overall survival of prostate cancer patients: A Surveillance, Epidemiology, and End Results (SEER) analysis. Can Urol Assoc J 2019;13:E135-9.

21. Robles TF, Slatcher RB, Trombello JM, et al. Marital quality and health: a meta-analytic review. Psychol Bull 2014;140:140-87.

22. Aragon-Ching JB. Adjuvant Chemotherapy for High-Risk Localized Prostate Cancer: Time for Change or Need More Time to Change? J Clin Oncol 2019;37:2296-7.

23. Rajan P, Sooriakumaran P, Nyberg T, et al. Effect of Comorbidity on Prostate Cancer-Specific Mortality: A Prospective Observational Study. J Clin Oncol 2017;35:3566-74.

24. Lavallée LT, Binette A, Witiuk K, et al. Reducing the Harm of Prostate Cancer Screening: Repeated ProstateSpecific Antigen Testing. Mayo Clin Proc 2016;91:17-22.

25. Thompson IM, Pauler DK, Goodman PJ, et al. Prevalence of prostate cancer among men with a prostate-specific antigen level $<$ or $=4.0 \mathrm{ng}$ per milliliter. $\mathrm{N}$ Engl J Med 2004;350:2239-46. 